\title{
FINANCIAL FRICTION AND MULTIPLICATIVE MARKOV MARKET GAMES
}

\author{
ERIK AURELL \\ Matematiska Institutionen \\ Stockholms Universitet, SE-106 91 Stockholm, Sweden \\ eaurell@matematik.su.se \\ PAOLO MURATORE-GINANNESCHI \\ CATS, The Niels Bohr Institute \\ Blegdamsvej 17, DK-2100 Copenhagen, Denmark \\ muratore@nbi.dk
}

\begin{abstract}
We study long-term growth-optimal strategies on a simple market with transaction costs. We show that several problems of this sort can be solved in closed form, and explicit the non-analytic dependance of optimal strategies and expected frictional losses of the friction parameter.
\end{abstract}

\section{Introduction}

The purpose of this paper is to present results on optimal control of investment strategies with friction. The discussion is for the most part couched in the frame-work of growth-optimal portfolios of underlying securities, the prices of which are smooth diffusions. We will show how to treat such problems in a Fokker-Planck framework, as an alternative to the Hamilton-Jacobi-Bellman equation methodology used in financial economics 10, 1], and explicit that several problems of this sort may be solved analytically in closed form. It appears clearly in the Fokker-Planck formulation that optimal strategies and expected friction lossses typically have a singular dependence on the friction parameter. For underlying smooth diffusions the friction losses scale with friction parameter to the the fractional power of two thirds. We consider also the more general situation, where the underlying is not a smooth diffusion, generally limiting ourselves to the discrete time case, and show how to relate to the optimal growth rate to the eigenvalue of the proper evolution operator. 
The subject matter of this paper is not new, and the result that optimal control of smooth diffusions with linear friction is singular is well known in control theory, see e.g. [6]. We believe that juxtaposing derivations in terms of FokkerPlanck and Hamilton-Jacobi-Bellman may help to make results obtained in the second language more accessible to physicists, who are typically more conversant with the first. On the other hand, the Fokker-Planck approach has a certain simplicity, and facilitates order-of-magnitude discussions and the use of a kind of dimensional analysis. An original motivation for this work was to derive an option pricing procedure by using growth-optimal criteria, generalizing work in the friction-less case [2, 3]. This is technically a harder problem. Various approximate solutions are possible and will be discussed in a forthcoming separate contribution [4].

\section{Stocks, bonds and transaction costs}

In this section we derive the dynamics of a stock and bond portfolio with proportional transaction costs in discrete time, and then take the continuum limit. We adhere to a representation of the dynamics given by a pre-hedging prescription. Other representations are possible, and give rise to dynamical equations, which although equivalent in content may superficially look different. A discussion of other prescriptions will be presented separately [4. We start by assuming that a source of of uncertainty is provided by the price movement of stock

$$
S_{t+\Delta t}=u_{t} S_{t}
$$

where time is discrete, $S_{t}$ is the price of a share at time $t$ and the $u_{t}$ 's are independent, identically distributed random variables. We also assume a riskless security (a bond or a bank account), with price movement

$$
B_{t+\Delta t}=r B_{t} \text {. }
$$

By a change of numeraire we can always take $r$ equal to one. We then assume proportional transaction costs. That is, an investor who holds some capital $C$ in stock or bonds at some time $t$, and would like to change his holding to the other security, would after the operation hold capital $(1-\gamma) C$, where $\gamma$ is a non-zero number. Several interpretations of $\gamma$ are possible. One is simply that there are direct fees coupled to every transaction. In real markets there are various fees, some proportional as we assume, some a fixed commission per transaction, and some with other structure such as different proportional commissions for buying and selling stock. For a real operator on a real market there are also indirect costs, such as overhead and operating costs, which, insofar as they can be determined from the outside, are perhaps roughly proportional to trading volume. Another interpretation of $\gamma$ is that there is really no one price, but only bid and ask prices, with some spread in between. If spread is proportional to price this will have the same effect as proportional friction.

We consider at time $t$ an investor who holds capital $W_{t}$, of which $\Delta W_{t}^{S}$ is invested in stock. The fraction invested in stock is hence $l_{t}=\frac{\Delta W_{t}^{S}}{W_{t}}$. The investor would like to change the amount invested in stock to $\alpha_{t} W_{t}$, and when rebalancing his portfolio he has to pay transaction costs of

$$
\Delta W_{t}^{T C}=\gamma\left|\alpha_{t} W_{t}-l_{t} W_{t}\right|
$$


The total wealth of the investor before the market moves again is hence $\left(1-\gamma \mid \alpha_{t}-l_{t}\right) W_{t}$, and after the market has moved at time $(t+\Delta t)$ the wealth and fraction of wealth invested in stock are

$$
\begin{aligned}
W_{(t+\Delta t)} & =\left(u_{t+\Delta t} \alpha_{t}+\left(1-\alpha_{t}\right)-\gamma\left|\alpha_{t}-l_{t}\right|\right) W_{t} \\
l_{(t+\Delta t)} & =\frac{u_{t+\Delta t} \alpha_{t}}{u_{(t+\Delta t)} \alpha_{t}+\left(1-\alpha_{t}\right)-\gamma\left|\alpha_{t}-l_{t}\right|}
\end{aligned}
$$

We see that the $l_{t}$ 's can be considered as consecutive positions in a series of gambles, or in a repeated game against Nature. The control variables $\alpha_{t}$ are on the other hand strategy choices made at different times (and in different positions) in the game. The game is multiplicative, since pay-off is proportional to capital, and it is Markov because the new capital and new position $\left(W_{(t+\Delta t)}\right.$ and $\left.l_{(t+\Delta t)}\right)$ depend parametrically on the previous position $\left(l_{t}\right)$. A simple special case is when transaction costs are zero and the dependence of wealth on previous position drops out. The game is then Bernoulli, and the only relevant variable is the strategy $\alpha_{t}$. The optimal strategies according to the growth-optimal criterion in this class of models were determined by Kelly 8 . For recent papers and reviews with references to the later literature, see e.g. [7, 9, 2, 3].

The continuum limit of (4) and (5) is reached by taking the random events to occur at times $0, d t, 2 d t, \ldots$, and modellling the stock price fluctuations by a stochastic differential

$$
u_{t}=1+\mu d t+\sigma d B_{t}
$$

$d B_{t}$ being increments of a Wiener process. Furthermore we assume that the control variable $\alpha_{t}$ is smoothly related to the position $l_{t}$ by

$$
\alpha_{t}=l_{t}+\beta_{t} d t
$$

The wealth fraction then changes as

$$
\begin{aligned}
& l_{(t+d t)}=l_{t}+\left[\left(\mu-\sigma^{2} l_{t}\right) l_{t}\left(1-l_{t}\right)+\beta_{t}+\gamma l_{t}\left|\beta_{t}\right|\right] d t+ \\
& {\left[\sigma l_{t}\left(1-l_{t}\right)\right] d B_{t}+\text { h.o.t }}
\end{aligned}
$$

where we have taken $d B_{t}$ to be formally of size $\sqrt{d t}$ and kept all terms up to linear in $d t$. Hence

$$
d l_{t}=\left(\left(\mu-\sigma^{2} l_{t}\right) l_{t}\left(1-l_{t}\right)+\beta_{t}+\gamma l_{t}\left|\beta_{t}\right|\right) d t+\left(\sigma l_{t}\left(1-l_{t}\right)\right) d B_{t}
$$

The wealth process can be written down in a similar fashion, after applying Ito's lemma in a form equivalent to

$$
d \log W_{t}=\left(\mu l_{t}-\frac{\sigma^{2}}{2} l_{t}^{2}-\gamma\left|\beta_{t}\right|\right) d t+\sigma l_{t} d B_{t}
$$

It is also useful for further reference to observe that without control the stochastic differential equation (9) preserves the three intervals $[-\infty, 0],[0,1]$ and $[1, \infty]$. A candidate for an optimal control will not move the process out of the best of 
the three intervals. Assuming for definiteness that the best interval is $[0,1]$ we introduce the variable $\xi=\log \frac{l}{1-l}$ and find

$$
d \xi_{t}=\left[\kappa+\mu-\frac{1}{2} \sigma^{2}\right] d t+\sigma d B_{t} \quad \kappa=\left(1+e^{\xi}\right)\left(\beta\left(1+e^{-\xi}\right)+\gamma\left|\beta_{t}\right|\right)
$$

The variable $\xi$ ranges from $-\infty$ to $+\infty$. Without control $(\beta$ and $\kappa$ equal to zero) $\xi$ follows a normal diffusion process with constant drift equal to $\mu-\frac{1}{2} \sigma^{2}$. For long times without control the process therefore ends up with $\xi$ far from the origin, that is $l$ close to zero or one. If the best intervals in $l$ are $[-\infty, 0]$ or $[1, \infty]$, the process without control in an analogous manner ends up in 0 or 1 , respectively. In other words, if you do not rehedge you eventually end with all your money in stock, or, depending on the parameters, with all your money in bonds.

\section{Long-term growth with transaction costs}

We start with some general remarks, borrowing some of the terminology from the theory of dynamical systems. Let us consider the dynamics of wealth and wealth fraction, where we write the strategy $\left(\alpha_{t}\right)$ as a function of position $\left(l_{t}\right)$ :

$$
\begin{aligned}
W_{(t+\Delta t)} & =\left(u_{(t+\Delta t)} \alpha_{t}\left(l_{t}\right)+\left(1-\alpha_{t}\left(l_{t}\right)\right)-\gamma\left|\alpha_{t}\left(l_{t}\right)-l_{t}\right|\right) W_{t} \\
l_{(t+\Delta t)} & =\frac{u_{(t+\Delta t)} \alpha_{t}\left(l_{t}\right)}{u_{(t+\Delta t)} \alpha_{t}\left(l_{t}\right)+\left(1-\alpha_{t}\left(l_{t}\right)\right)-\gamma\left|\alpha_{t}\left(l_{t}\right)-l_{t}\right|}
\end{aligned}
$$

The dynamics in $l$ induces a linear evolution equation for the probability distribution over the states in the corresponding Markov chain (i.e., the values of $l$ ). The asymptotic distribution in $l$ is the eigenvector corresponding to the highest eigenvalue of the corresponding operator, let that be written $P_{0}(l ;[\alpha]) d l$. The long-term growth rate is thus

$$
\lambda(\gamma ;[\alpha])=\int P_{0}(l ;[\alpha]) E[\log (1+\alpha(l)(u-1)-\gamma|\alpha(l)-l|)] d l
$$

the expected logarithmic return with respect to the joint distribution over $l$ and the random variable $u$ 円 Maximizing long-term growth is a non-trivial problem because the strategy $\alpha$ enters both in the function to be averaged, i.e. $\log (1+\alpha(l)(u-1)-\gamma|\alpha(l)-l|)$, and in the measure of integration.

To proceed further we turn to the continuum limit, as defined by equations (9) and (10). We will consider a model problem, and show how that can be solved by Fokker-Planck methods. Then we will repeat the model problem in the Hamilton-Jacobi-Bellman formalism, and then return to the full problem of equations (9) and (10). A kind of dimensional analysis and possible extentions of the procedure are discussed separately in section 4 .

\footnotetext{
${ }^{1}$ In our model $l$ and $u$ are independent, so the joint distribution is the product of the distributions over $l$ and $u$ separately. In a proper game one would expect that the $u$ would not be independent of $l$ - and also dependent on the positions of the other players in the game.
} 


\subsection{A model problem}

Let us consider the stochastic differential equation

$$
d x_{t}=-\left[\frac{\partial V}{\partial x}\right] d t+\sqrt{2 D} d B_{t}
$$

and suppose that we wish to maximize

$$
U=E\left[a x-\frac{1}{2} b x^{2}-c\left|\frac{\partial V}{\partial x}\right|\right]
$$

where the expectation value is taken with respect to the stationary distribution of $x$,

$$
E[f(x)]=\int N^{-1} e^{-\frac{V(x)}{D}} f(x) d x
$$

The normalization factor $N$ is fixed by $E[1]=1$. The problem is hence to maximize with Lagrange multiplier $q$ the functional

$$
Q=N^{-1} \int e^{-\frac{V(x)}{D}}\left(a x-\frac{1}{2} b x^{2}-c\left|\frac{\partial V}{\partial x}\right|\right) d x+q\left(\int e^{-\frac{V(x)}{D}} d x-N\right)
$$

We vary with respect to $V^{\prime}(x)$ to find

$$
\begin{aligned}
\frac{\delta Q}{\delta V^{\prime}(x)}= & N^{-1} \int_{x}^{\infty} e^{-\frac{V(x)}{D}}\left(-\frac{1}{D}\right)\left(a x-\frac{1}{2} b x^{2}-c\left|\frac{\partial V}{\partial x}\right|+q N\right) d x \\
& -N^{-1} c \operatorname{sign}\left(\frac{\partial V}{\partial x}\right)
\end{aligned}
$$

At an extremum we have $\frac{\delta Q}{\delta V^{\prime}(x)}=0$, for every $x$ such that $V(x)<\infty$. If we differentiate with respect to $x$ two terms cancel, and we have the simpler equation

$$
a x-\frac{1}{2} b x^{2}+q N-2 D c \delta\left(V^{\prime}(x)\right) V^{\prime \prime}(x)=0 .
$$

Clearly it is not possible to satisfy (20) unless $V^{\prime}=0$. If $V^{\prime}=0$ the equation can be understood in the distributional sense, for instance by broadening the $\delta$ function to be $1 / \epsilon$ on the interval $[-\epsilon / 2, \epsilon / 2]$, and zero elsewhere. Then (20) is equivalent to the ordinary differential equation $V^{\prime \prime}=\frac{\epsilon}{2 D c}\left(a x-\frac{1}{2} b x^{2}+q N\right)$ as long as $V^{\prime}$, the integral of $V^{\prime \prime}$, is not larger than $\epsilon / 2$ in absolute value. This equation can obviously be satisfied on an interval in $x$ of finite width. To find the optimal interval without making an explicit assumption on the regularization, we just assume that $V$ is large outside an interval $\left[x_{-}, x_{+}\right]$and that $V^{\prime}$ is small in the same interval. The quantity to maximize can then be made arbitrary close to

$$
U\left(x_{-}, x_{+}\right)=\frac{a}{2} \frac{\left(x_{+}\right)^{2}-\left(x_{-}\right)^{2}}{x_{+}-x_{-}}-\frac{b}{6} \frac{\left(x_{+}\right)^{3}-\left(x_{-}\right)^{3}}{x_{+}-x_{-}}-\frac{2 D c}{x_{+}-x_{-}}
$$


which is maximized by

$$
x_{ \pm}=\frac{a}{b} \pm \frac{1}{2}\left(\frac{24 c D}{b}\right)^{\frac{1}{3}}
$$

The optimum control is thus given by a "deep-pit" potential with mid-point $\frac{a}{b}$ and width $\left(\frac{24 c D}{b}\right)^{\frac{1}{3}}$. The value of the maximum is

$$
U_{\max }=\frac{a^{2}}{2 b}-\frac{1}{2}\left(9 b D^{2} c^{2}\right)^{\frac{1}{3}}
$$

The loss of utility is therefore proportional to friction parameter $c$ to the power two thirds.

\subsection{Hamilton-Jacobi-Bellman}

Dynamical programming is formulated in the setting that we have a SDE

$$
d \xi_{t}=f\left(\xi_{t}, u\right) d t+\sigma\left(\xi_{t}, u\right) d B_{t}, \quad \xi_{s}=x
$$

where $u=u(x, t)$ is a dynamic control function. We wish to extremize a functional, generally of a form like

$$
J(x, s)=\inf _{u \in \Re}\left\{E_{\xi_{s}=x} \int_{s}^{T} d t L\left(\xi_{t}, D^{+} \xi_{t}\right)+J\left(\xi_{T}, T\right)\right\}
$$

where the forward derivative is defined as

$$
D^{+} F\left(\xi_{t}\right)=\lim _{h \downarrow 0} E_{\xi_{s}=x}\left\{\frac{F\left(\xi_{t+h}, t+h\right)-F(x, t)}{h}\right\} .
$$

The solutions of (24) are assumed to be smooth diffusions for any admissible choice of $u$. $\mathrm{n}$ our model problem there is no control of the diffusion term, i.e. $\sigma\left(\xi_{t}, u\right)=\sqrt{2 D}$ does not depend on $u$. The drift term $f\left(\xi_{t}, u\right)$ is on the other hand $-\frac{\partial V}{\partial x}$, and we may also identify $D^{+} \xi_{t}$ with $f$. Hence, if $L\left(\xi_{t}, D^{+} \xi_{t}\right)=-a \xi_{t}+\frac{b}{2} \xi_{t}^{2}+c\left|D^{+} \xi_{t}\right|$ and $J\left(\xi_{T}, T\right)=0$, then $\frac{J(x, s)}{T-s}$ is the minimum of the average value of the functional $L$ which can be achieved in the time interval $[s, T]$, given that the process starts in point $x$ at time $s$. If the process (24) is stationary, then the large $T$ limit of this average will be almost surely opposite in sign but equal in absolute value of the expectation value to be maximized in (16).

The idea is to recast the problem in terms of a differential equation. Bellman's principle states that for any $h$ between 0 and $T-s$

$$
J(x, s)=\inf _{u \in \Re}\left\{E_{\xi_{s}=x} \int_{s}^{s+h} d t L\left(\xi_{t}, D^{+} \xi_{t}\right)+J\left(\xi_{s+h}, s+h\right)\right\}
$$


With the assumptions that the process (24) is a smooth diffusion (already made), and that $J(x, s)$ is a smooth function of $x$ and $s$ (which follows if (24) is a smooth diffusion and $s$ less than $T$ ), we have

$$
\begin{gathered}
0=\lim _{h \downarrow 0} \inf _{u \in \Re} E_{\xi_{s}=x}\left\{\frac{1}{h} \int_{s}^{s+h} d t L\left(\xi_{t}, D^{+} \xi_{t}\right)+\frac{J\left(\xi_{s+h}, s+h\right)-J(x, s)}{h}\right\}= \\
=\inf _{u \in \Re}\left\{L(x, u)+\partial_{s}+f(x, u) \partial_{x}+\frac{\sigma(x, u)^{2}}{2} \partial_{x}^{2}\right\} J(x, s)
\end{gathered}
$$

Substituting $L, \sigma\left(\xi_{t}, u\right)$ and introducing $\lambda=-J$ we have

$$
\begin{aligned}
& \partial_{s} \lambda+\sup _{f}\left\{f \partial_{x} \lambda+D \partial_{x}^{2} \lambda+a x-\frac{b}{2}-c|f|\right\}=0 \\
& \lambda(x, T ; T)=0
\end{aligned}
$$

Naive variation with respect to $f$ leads to $\partial_{x} \lambda=c \operatorname{sign}(f)$. This is problematical because the sign function is not invertable, and we therefore have no equation for the control. Furthermore, control entirely drops out of (29), and we have instead the much simpler equation $\partial_{s} \lambda+D \partial_{x}^{2} \lambda+a x-\frac{b}{2}=0$, with solution $\lambda(x, s)=(T-s)\left(a x-\frac{b}{2} x^{2}\right)-\frac{D b(T-s)^{2}}{2}$. The average $\lambda /(T-s)$ decreases asymptotically linearly as $-D b(T-s) / 2$ with increasing $T$. In fact, all we have solved for is the expected value of $a x-\frac{b}{2} x^{2}$ of an uncontrolled random walk with diffusion coefficient $D$ starting from $x$ and moving for a time $T-s$.

The problem is that motion in a "deep-pit" potential, which we already know is the asymptotically optimal control, is not a smooth diffusion at the boundary. For a full discussion, see e.g. [6], to extend (slightly) the Hamilton-JacobiBellman in a less rigorous manner, assume an interval $\left[x_{-}, x_{+}\right]$such that the restoring force $f$ goes to positive and negative infinity, respectively, when $x$ tends to the boundary points $x_{-}$and $x_{+}$from the inside. Outside the interval $f$ is supposed to remain infinite, so that the process is constrained to be in $\left[x_{-}, x_{+}\right]$. If further the variational equation $\partial_{x} \lambda=c \operatorname{sign}(f)$ is interpreted in the distributional sense, for instance by broading the sign function to tanh $\frac{f}{\epsilon D}$, then $f$ is determined as a function of $\partial_{x} \lambda$ inside the interval, of size $\epsilon$ away from the boundaries, and $\lambda$ satisfies the boundary conditions

$$
\left.\partial_{x} \lambda\right|_{x=x_{-}}=\left(-\left.\partial_{x} \lambda\right|_{x=x_{+}}\right)=c
$$

The dynamic programming equation (29) can now be solved for large $T$, if we make the ansatz

$$
\lambda(x, s ; T) \rightarrow \frac{(T-s)}{\tau}+\text { asymptotically constant in } T
$$

When (31) holds true one integration in $x$ in (29) gives $D \partial_{x} \lambda=A+\frac{1}{\tau} x-\frac{a}{2} x^{2}+$ $\frac{b x^{3}}{6}$ and the two boundary conditions read

$$
A+\frac{1}{\tau} x_{-}-\frac{a}{2} x_{-}^{2}+\frac{b}{6} x_{-}^{3}=-\left(A+\frac{1}{\tau} x_{+}-\frac{a}{2} x_{+}^{2}+\frac{b}{6} x_{+}^{3}\right)=D c
$$


which are easily separated into one equation for the constant of integration $A$, and one equation for $\tau$, which is identical to (21). We have therefore derived the same result again in the HJB formalism. As a consistency check for the solution one verifies that in the interval $\left[x_{-}, x_{+}\right]$the absolute value of the gradient of $\lambda$ is bounded by $c$.

\subsection{The full stock and bond problem}

It is convenient to consider the problem in terms of the transformed variable $\xi$ of (11), which obeys a stochastic differential equation entirely similar to the model problem 15) with $-\partial_{x} V=\left(\kappa+\mu-\sigma^{2} / 2\right)$ and $D=\sigma^{2} / 2$, but where the function to maximize is

$$
F=E\left[\mu\left(\frac{e^{\xi}}{1+e^{\xi}}\right)-\frac{1}{2} \sigma^{2}\left(\frac{e^{\xi}}{\left(1+e^{\xi}\right)^{2}}\right)-\frac{e^{\xi}}{1+e^{\xi}} \frac{\gamma|\kappa|}{1+e^{\xi}(1+\gamma \operatorname{sign}(\kappa))}\right]
$$

In deriving (33) we have expressed the control $\beta$ (of $l$ ) in terms of the control $\kappa$ (of $\xi$ ). Let us write $F=E\left[\left.g(x, u)\right|_{u=\partial_{x} V}\right]$. The equation analogous to (20) will then be

$$
\left.\frac{\partial^{2} V}{\partial x^{2}} \frac{d^{2} g}{d u^{2}}\right|_{u=\partial_{x} V}-\left.\frac{1}{D} \frac{\partial V}{\partial x} \frac{d g}{d u}\right|_{u=\partial_{x} V}+\left.\frac{d^{2} g}{d x d u}\right|_{u=\partial_{x} V}+\frac{1}{D}(g-q N)=0
$$

This equation will not have solutions (when $V<\infty$ ) unless $\kappa$ vanishes. The invariant density is hence

$$
P(\xi)=N^{-1} \exp \left(\xi \frac{\mu-\sigma^{2} / 2}{\sigma^{2} / 2}\right) \quad \xi \in\left[\xi_{-}, \xi_{+}\right]
$$

and the two endpoints $\xi_{-}$and $\xi_{+}$can be determined by maximizing

$$
\begin{aligned}
F\left(\xi_{-}, \xi_{+}\right)= & N^{-1} \int_{\xi_{-}}^{\xi_{+}} e^{\left(\frac{2 \mu}{\sigma^{2}}-1\right) \xi}\left(\mu\left(\frac{e^{\xi}}{1+e^{\xi}}\right)-\frac{1}{2} \sigma^{2}\left(\frac{e^{\xi}}{\left(1+e^{\xi}\right)^{2}}\right)\right) d \xi \\
& -\gamma \frac{\sigma^{2}}{2} \frac{1}{N}\left(e^{\left(\frac{2 \mu}{\sigma^{2}}-1\right) \xi} \frac{e^{\xi_{-}}}{1+e^{\xi_{-}}} \frac{1}{1+e^{\xi_{-}}(1+\gamma)}\right) \\
& -\gamma \frac{\sigma^{2}}{2} \frac{1}{N}\left(e^{\left(\frac{2 \mu}{\sigma^{2}}-1\right) \xi_{+}} \frac{e^{\xi_{+}}}{1+e^{\xi_{+}}} \frac{1}{1+e^{\xi_{+}}(1-\gamma)}\right)
\end{aligned}
$$

In the small $\gamma$ limit it is sufficient to observe that the optimal interval is centered on $l=\frac{\mu}{\sigma^{2}}$, and that the width is determined by (22) with $D=\frac{\sigma^{2}}{2}\left(\frac{\mu}{\sigma^{2}}\right)^{2}\left(1-\frac{\mu}{\sigma^{2}}\right)^{2}$, $b=\sigma^{2}$ and $c=\gamma$. Inserting these quantities we have

$$
\Delta l \approx\left(12 \gamma\left(\frac{\mu}{\sigma^{2}}\right)^{2}\left(1-\frac{\mu}{\sigma^{2}}\right)^{2}\right)^{\frac{1}{3}} \quad \gamma \text { small }
$$

When $\mu / \sigma^{2}$ is close to zero or one the width of the holding interval vanishes. This is natural, since if the optimal investment is to hold all money in stock or bonds no rehedging is necessary. On the other hand, when $\mu / \sigma^{2}$ is around 
one half the optimal holding interval is about $0.9 \gamma^{\frac{1}{3}}$. For realistic values of $\gamma$ this is not a small number. For instance, if $\gamma$ is one percent, and the best portfolio without friction is to hold half each in stock and bonds, then the optimal strategy is to not rehedge unless the fraction in stock falls below 40 or rises above 60 percent.

\section{Dimensional analysis}

It is instructive to see that most of the results can be derived by a kind of dimensional analysis. Let us consider the model problem, and call the dimension of the quantity to maximize $U$. In the full problem it would be wealth per time. Let $L$ be the dimension of the quantity $x$. The dimensions of the parameters are then

$$
[a]=\frac{U}{L} \quad[b]=\frac{U}{L^{2}} \quad[c]=\frac{U T}{L} \quad[D]=\frac{L^{2}}{T}
$$

From $a$ and $b$ we can form one quantity with dimension $L$, namely $a / b$. This is the optimal value of $x$ without friction. From $b, D$ and $c$ we can also form one quantity with dimension $L,(D c / b)^{\frac{1}{3}}$, which, up the numerical prefactor, is indeed the width of the holding interval.

From $D$ and the width of the holding interval $(d)$ we can form a characteristic time between rehedgings $\tau=d^{2} / D$. If the growth-optimal criterion is to have a special significance for an individual investor we must suppose that his investment horizon is much longer than $\tau$. For realistic parameter values this implies surprisingly long time scales. If we use the previously discussed example with $\gamma=0.01$, and assume $\sigma$ one percent per day, then the characteristic time is 800 days, about three years of trading time. If we assume instead the extreme and probably unrealistic value of $\gamma$ one part in a million, then the holding interval is still one percent in width, and the characteristic time is measured in days. The conclusion is, that in the very simple model of one stock and one bond, with transaction costs, it is delicate and rather doubtful if times are ever sufficiently long that the growth-optimal criterion is unquestionably valid. If we enlarge the model to include more securities, especially derivative securities the return distributions of which depend explicitly on time to maturity, this conclusion can only be strengthened. For the growth-optimal criterion to be of special significance one would have to discuss not individual investors, but evolving populations of investment strategies, and to show, if true, that growthoptimal strategies in multiplicative games approximate non-cooperative Nash equilibria, selected by the Nash "mass-action" principle 11. For a recent discussion of populations of evolving strategies, see also [5].

We can also use dimensional analysis to consider sources of uncertainty other than smooth diffusions. If a noise term $d w_{t}$ is formally taken to be $(d t)^{\alpha}$, then $D$ will be of dimension $L^{2} / T^{2 \alpha}$, the characteristic width $d=c^{\frac{\alpha}{1+\alpha}} D^{\frac{1}{2+2 \alpha}} b^{-\frac{\alpha}{1+\alpha}}$ and $\tau=\left(\frac{d^{2}}{D}\right)^{\frac{1}{2 \alpha}}$. The expected friction losses per unit time are $c d / \tau$. A simple strategy with this scaling is to bring back the portfolio to the optimum (cost $c d$ ) every time the fraction goes out of a band of width $d$ (event occuring with frequency $1 / \tau)$. For smooth diffusion this is the same scaling as in (23); only the 
prefactor will be better with the optimal strategy. Since for realistic parameter values the characteristic times are typically long, it however probably suffices that the noise is Gaussian and uncorrelated on those time scales for the optimal control of smooth diffusions that have mainly been discussed here to be relevant.

\section{Acknowledgments}

We thank P. Dimon, S. Ghirlanda, O. Hammarlid, M. van Hecke, A. Kozlov, G. Lacorata, A. Martin-Löf, G. Smirnov and K. Życzkowski for discussions. E.A. thanks Prof. S.M. Gama and the Departamento do Matematica Aplicada of Universidade do Porto for hospitality. We thank the organizers of European Dynamics Days 1999 (Como June 20-23, 1999) and the European Physical Society Conference "Applications of Physics to Financial Analysis" (Dublin July 14-17, 1999) for opportunities to present this work, and the Swedish Natural Research Council and the European Community for financial support under grants M-AA/FU/MA 01778-333 (E.A.) and ERB4001GT962476 (P.M.-G.).

\section{References}

[1] M. Akian, J-L. Menaldi and A. Sulem, "On an investment-consumption model with transaction costs", SIAM J. Control and Optim., 34 (1996) 329-364.

[2] E. Aurell, R. Baviera, O. Hammarlid, M. Serva and A. Vulpiani, (1998), "A general methodology to price and hedge derivatives in incomplete markets", IJTAF [in press].

[3] E. Aurell, R. Baviera, O. Hammarlid, M. Serva and A. Vulpiani, "Gambling and Pricing of Derivatives". (1998). Social Science Research Network Electronic Library (http://papers.ssrn.com/.

[4] E. Aurell and P. Muratore-Ginanneschi (in preparation).

[5] J.Doyne Farmer "Market force, ecology, and evolution", Los Alamos e-Print Archive adap-org/9812005.

[6] W.H.Fleming and H.Mete Soner Controlled Markov Processes and Viscosity Solutions (Springer-Verlag Berlin, 1992).

[7] N. Hakanson and W. Ziemba, "Capital Growth Theory", in Handbooks in OR $\mathscr{G}$ MS, Vol.9, eds. R. Jarrow et al. (Elsevier Science, 1995).

[8] Jr. J. L. Kelly, "A new interpretation of the Information Rate", Bell Syst. Tech. J. 35 (1956) 917.

[9] S. Maslov and Y.-C. Zhang, "Optimal Investment Strategy for Risky Assets", International J. of Theoretical and Applied Finance 1 (1998) 377-388.

[10] R. Merton, Continuous-Time Finance (Blackwell, 1990).

[11] J. Nash, Non-cooperative games, Ph.D. thesis (Princeton University, 1950). 